\title{
Construcción participativa del regionalismo estratégico: ¿hacia una agenda medioambiental externa del Mercosur?
}

Participatory construction of strategic regionalism: towards an external environmental agenda of Mercosur?

Rev. Bras. Polít. Int. 55 (1): 194-210 [2012]

\section{Introducción}

La década de los noventa del siglo XX, signada por el avance del proceso de globalización y por el llamado regionalismo abierto, conjugó un marco de acción diferente para los países de América Latina y el Caribe (ALC). Contextualmente fue determinante la influencia creciente de las relaciones externas sobre el diseño de las políticas internas. A la inversa, los países en vías de desarrollo veían debilitada su capacidad de incidencia en foros internacionales relevantes. Resaltaban aquí dos cuestiones (Bizzozero 2011a, 5). A nivel de las relaciones internacionales, y en función de los recursos de poder, resultan cruciales las respectivas capacidades para impulsar o participar en la definición de los regímenes internacionales tendientes a delinear un nuevo orden. Por otra parte, se planteaba a los Estados la cuestión relativa a la economía y la política de desarrollo, con una agenda crecientemente interméstica.

Para América Latina y el Caribe (ALC) se tornó crítico alcanzar suficiente cohesión en su proyección externa: la entidad regional debía explicitarse en un mundo donde interactuaban actores con capacidad de acción global, grupos regionales de Estados y entidades transnacionales. En el Mercosur, y en razón del objetivo de la Unión Aduanera, la articulación de la política comercial común y la participación conjunta en negociaciones internacionales concentraron gran parte de los esfuerzos. En contraste, la preocupación por las políticas de desarrollo sustentable en los países del bloque y su articulación externa se esboza con una

\footnotetext{
* Investigadora del Sistema Nacional de Investigadores (SNI) de la Agencia Nacional de Investigación e Innovación y Docente del Diploma en Estudios Internacionales de la Universidad de la República (UDELAR), Uruguay (astuhldreher@hotmail.com).
} 
mención genérica en el Tratado de Asunción. Sin embargo, la Declaración de Canela (1992) así como la Declaración de Taranco (1995) denotan esfuerzos tempranos en la búsqueda de posicionamientos medioambientales conjuntos, que aunque fueron menos consecuentes dieron lugar a iniciativas diversas.

Ante la proximidad de Río+20, el presente artículo tematiza en primer término hitos relevantes de la búsqueda de posicionamientos conjuntos del bloque en materia medioambiental. En segundo lugar, se contextualiza dicha búsqueda en el marco de la evolución reciente del proceso regional. En tercer lugar, partiendo de la referencia incluida en diversos tratados internacionales acerca de la participación pública como presupuesto del desarrollo sostenible, se argumenta desde el enfoque del constructivismo social como marco interpretativo del aporte de las comunidades epistémicas y de la sociedad civil para definir una agenda medioambiental. En cuarto lugar se consideran los casos de Brasil y Argentina. Finalmente, considerando logros y déficits, se esbozan algunas perspectivas a futuro.

\section{Mercosur: búsqueda de posiciones medioambientales conjuntas}

La cuestión del desarrollo sostenible fue esbozada en el Tratado de Asunción al mencionar la meta del desarrollo económico con justicia social: «(...) ese objetivo debe ser alcanzado mediante el más eficaz aprovechamiento de los recursos disponibles, la preservación del medio ambiente, el mejoramiento de las interconexiones físicas, la coordinación de las políticas macroeconómicas y la complementación de los diferentes sectores de la economía con base en los principios de gradualidad, flexibilidad y equilibrio» (Mercado Común del Sur 1991). Por su alcance regional el acuerdo debía abarcar necesariamente la cuestión ambiental, generando normas vinculadas directa o indirectamente con la temática y que favorecieran la armonización en la materia. ${ }^{1}$

Respecto a la proyección externa, la Declaración de Canela de febrero de 1992 de los presidentes del Cono Sur y de Chile procuró definir una posición común sobre temas ambientales, proclamando el compromiso con el desarrollo sostenible y la responsabilidad en materia de protección ambiental y el uso racional de los recursos naturales, postulando la necesidad de cooperación científica y tecnológica con los países desarrollados. Como principio medular regía la complementariedad entre la protección del medioambiente y el desarrollo económico.

Por otra parte, la Resolución del Grupo Mercado Común (GMC) No 10/94, que define las Directrices Básicas en Materia de Política Ambiental para el Mercosur, establece la coordinación de criterios ambientales comunes para la negociación del bloque a nivel internacional, mientras que la Declaración de

1 Sobre la evolución institucional y legal medioambiental en el Mercosur véase Secretaría Mercosur (2006) y Martínez Arteaga (2011, 88 y ss.). 
Taranco de 1995 señala el compromiso de coordinación de posiciones conjuntas en foros mundiales de medioambiente (en particular biodiversidad, cambio climático y Comisión de desarrollo sustentable), tematizando la implementación de la Agenda 21 y los acuerdos ambientales multilaterales (Colacrai 1998, 307-308; Martínez Arteaga 2011, 95). La Decisión 9/95 del Consejo del Mercado Común (CMC) aprueba el «Programa de Acción del Mercosur hasta el año 2000», planteando una planificación estratégica que retoma el concepto de la promoción del desarrollo económico regional sustentable con justicia social coincidente con la conceptualización de Naciones Unidas.

Pese a esto, las dificultades de articulación se hicieron evidentes al no consensuarse el Protocolo Adicional al Tratado de Asunción sobre Medio Ambiente, que no fue apoyado por Argentina. La propuesta recogía postulados de la Declaración de Río de 1992, como la internalización de los costos ambientales en los procesos productivos de bienes y servicios, así como el pago por el uso de recursos naturales. ${ }^{2}$ En 2001, por medio de una Decisión del CMC se aprueba un Acuerdo Marco sobre Medio Ambiente, cuyo Preámbulo explicita el objetivo del desarrollo económico social y ambientalmente sustentable. Asimismo se fija el compromiso de los Estados partes a cumplimentar los acuerdos internacionales en materia ambiental, se ratifican los principios de la Declaración de Río y de la Agenda 21 y la complementariedad entre políticas comerciales y medioambientales.

En 2009 en el marco de la X Reunión de Ministros de Medio Ambiente se propusieron lineamientos para un proyecto de cooperación de adaptación al cambio climático en el Mercosur. Más allá de las posibles estrategias coordinadas de adaptación, se apuntó a lograr cooperación financiera internacional, transferencia tecnológica y creación de capacidades de acuerdo a las necesidades de los países de la región. De cara a la Conferencia de Naciones Unidas para el Desarrollo Sostenible Río+20, durante la XI Reunión XI de Ministros de Medio Ambiente se expresó la intención de plasmar conjuntamente los intereses y desafíos del Mercosur, permitiendo una reflexión conjunta sobre la aspiración de la región en términos medioambientales y los caminos críticas para su realización. Se acordó redactar un documento común sobre los avances desde Río 1992, así como incentivar a los movimientos sociales a aportar propuestas. Finalmente, durante la XI Reunión en noviembre de 2010 en Brasilia, se discutió la posición conjunta para la Cumbre de Cancún sobre Cambio Climático. ${ }^{3}$ En relación a Río+20 se acordó realizar un balance de los avances desde Río 1992 y revisar los enfoques del Mercosur sobre «economía verde».

2 Dado el mayor grado de avance de la legislación brasilera de protección ambiental, Argentina temía los impactos a nivel de competitividad de una rápida incorporación del Protocolo. También preocupaba la falta de armonización de los estándares ambientales.

3 El documento expresa preocupación sobre la experiencia de Copenhague, donde los países de la región no consiguieron articular una posición conjunta. Se tematizan las características que la cooperación internacional sobre cambio climático debería adoptar para satisfacer las necesidades de la región (Secretaría de Medioambiente 2010). 


\section{Evolución reciente del Mercosur}

Para enmarcar la búsqueda de posicionamientos conjuntos en el Mercosur respecto al desarrollo medioambientalmente sustentable, cabe visualizar el devenir del proceso regional durante la última década, que desde la teoría política de la integración regional fue conceptualizado como regionalismo estratégico (Briceño Ruiz 2006; Guerra Borges 2009; Bizzozero 2009; 2011a; 2011b). Con el inicio del siglo XXI se dan cambios de gobierno en la región, coincidiendo con la revisión de las prioridades del modelo de desarrollo brasilero y de su política exterior hacia la región, fortaleciendo progresivamente la noción del Mercosur como opción estratégica para mejorar la inserción internacional. Junto a la discusión sobre los vínculos del regionalismo con la reestructuración del orden internacional, se procura visualizar el rol de los llamados emergentes del sistema, concretamente las potencias medias y regionales como Brasil, para determinar su capacidad de incidir activamente en la definición de diversos issues en diferentes niveles de la estructura internacional (Bizzozero 2011a, 36 ss.).

Respecto a los modelos de desarrollo, cabe recordar que una cuestión ya planteada en el debate en torno de los «nuevos regionalismos» de los noventa se refería a si efectivamente conformaban marcos de articulación de ideas y del ámbito normativo-institucional, mediando entre el proceso de globalización y los procesos nacionales. Con el denominado regionalismo estratégico y la redefinición de prioridades temáticas, surgen nuevos orientadores simbólicos, reforzándose la idea de que el Mercosur no constituye simplemente un bloque comercial sino también un espacio catalizador de valores, tradiciones y futuro compartido (Bizzozero 2011a, 7). Resultan ilustrativos los lineamientos del Consenso de Buenos Aires de octubre de 2003, del documento de Río de Janeiro y del Acta de Copacabana de 2004, así como del Programa de Trabajo 2004-2006, que incluyeron temas pendientes desde los noventa y que pueden ser englobados bajo el rótulo de «crecimiento con equidad».

La ausencia de pronunciamientos explícitos en materia medioambiental es indicativa de que todavía no se visualizaba a los desafíos medioambientales como centrales para el nuevo impulso que se pretendía dar al proceso regional. Así, en el Programa de Trabajo 2004-2006 que define áreas específicas (económicocomercial, social, institucional) no se hace mención expresa ni transversal de las connotaciones medioambientales de cada una de esas áreas, así como tampoco incluye a la temática en la nueva agenda del bloque, donde se focalizan los programa de cooperación en ciencia y tecnología así como la integración física y energética (Mercosur/CMC/Dec. No 26/03). Se evidencia así la debilidad en la articulación de las políticas ambientales de los países miembros con las políticas económicas y sociales respectivas, debilidad que se traslada a los lineamientos diseñados en clave estratégica para el bloque. La disociación temática es especialmente llamativa 
después de que por Decisión del CMC No 02/01 en $2001^{4}$ se hubiera aprobado el ya mencionado Acuerdo Marco sobre Medio Ambiente del Mercosur y después de los debates de la Cumbre Internacional sobre la Financiación del Desarrollo en Monterrey y de la Cumbre Mundial de Desarrollo Sostenible en Johannesburgo en 2002, que señalaban la interrelación entre desarrollo, pobreza y medioambiente y enfatizaban la necesidad de coherencia de las políticas internas.

\section{Construcción participativa de la agenda Mercosur: aportes del enfoque constructivista}

El planteamiento de nuevas cuestiones en el marco del «regionalismo estratégico» favoreció a su vez una nueva modalidad de participación de actores en la región, que asumiendo determinados roles en la configuración del entramado institucional influyeron en el devenir del proceso, ya que la estructura institucional otorga competencias a actores gubernamentales y no gubernamentales, tales como académicos, científicos y expertos de organizaciones de la sociedad civil, generando efectos de derrame sociopolítico (Bizzozero 2011b, 7-8). Destacable resulta el hecho que aunque el impulso para esta "construcción» provendría de la institucionalidad del Mercosur, con fuerte inducción desde los gobiernos, la misma sería reapropiada desde lo regional-nacional para ser reconducido a las instancias nacionales donde se materializa una horizontalidad intrasocial que devendría intersocial y proporcionaría eventualmente insumos para el desarrollo de una identidad regional. Según esta interpretación, la evolución muestra un lento proceso de construcción de principios, normas y valores con participación de sectores diversos de la sociedad civil, académicos y políticos, con resultados específicos que en algunos casos están relacionados con bienes públicos regionales.

La cuestión de la participación resulta relevante al momento de dilucidar la capacidad del bloque de darse un ordenamiento medioambiental que pueda servir de base para la elaboración de una agenda externa concomitante. Una mirada a la situación de los países miembros puede proporcionar algunas claves de interpretación según los postulados teóricos del constructivismo social como expresión de las teorías basadas en el conocimiento. En el marco del así llamado «tercer debate» en la disciplina de las relaciones internacionales, los llamados pospositivistas parten del supuesto que la realidad social es construida socialmente y que por lo tanto no puede ser interpretada en forma inconexa del individuo y su contexto. En oposición a los enfoques positivistas que parten de actores

4 El Acuerdo establece criterios orientadores en materia ambiental. El Preámbulo plantea el objetivo para la región de lograr un desarrollo económico, social y ambientalmente sustentable, estableciendo el compromiso de las partes de cooperar para cumplimentar acuerdos internacionales en materia ambiental. Se reafirman los principios de la Declaración de Río de 1992 y la Agenda 21, subrayándose la complementariedad entre políticas comerciales y ambientales. 
racionales con esquemas de preferencias fijas (homo oeconomicus), para los enfoques pospositivistas las identidades, intereses y preferencias de los actores no son determinadas en forma exógena, sino que se configuran en un proceso endógeno (homo sociologicus) (Hänggi 1998).

Dentro del constructivismo social con su énfasis en el rol central de las identidades colectivas como determinante de los intereses estatales, se destaca la interpretación de Wendt respecto a que la identidad social del Estado se conforma exclusivamente a partir de la interacción con otros estados y con las estructuras del sistema internacional. Consecuentemente, la estructura anárquica del sistema internacional no necesariamente conduce al imperio de la lógica de un sistema de autoayuda orientado según consideraciones de poder, lo que posibilita prácticas interestatales que a través de procesos de aprendizaje induzcan un cambio en las relaciones internacionales (Wendt 1992).

Aunque la referencia a la constitución endógena de las identidades introdujo un elemento dinámico en las explicaciones hasta ahora estáticas de enfoques teóricos sistémicos neorrealistas o institucionalistas, el aporte de Wendt también parte del nivel «sistema internacional», relativizando la dimensión intraestatal. Sin embargo, en sentido estricto, el constructivismo social asume una posición analítica-ontológica intermedia entre actor y estructura, por lo que la explicación de Koslowski y Kratochwil surge como más plausible en términos de explicación del cambio en las relaciones internacionales (Koslowski y Kratochwil 1994, 216). Este ocurriría cuando los actores a través de sus prácticas modifican las reglas y normas constitutivas de la interacción internacional. La misma reproducción de la práctica de actores internacionales (como los Estados) depende de la reproducción de prácticas de actores nacionales (individuos o grupos). En consecuencia, los cambios fundamentales en política internacional se darían cuando las creencias y las identidades de los actores nacionales cambian, alterando de esa forma las reglas y normas constitutivas de sus prácticas políticas. Así, es el nivel de la sociedad (en donde se construyen las identidades colectivas) el que en definitiva es responsable de la definición de los intereses estatales y de la praxis de cada Estado en la esfera de las relaciones internacionales: el cambio de las identidades colectivas se produce cuando se han transformado las ideas que le sirven de base.

En lo que hace a la transmisión de ideas, un actor de importancia indiscutible son las comunidades epistémicas. Según Haas (1992), dichas comunidades constituyen grupos nacionales o transnacionales de profesionales que comparten determinada visión del mundo (episteme): disponen de reconocida expertise en un área en particular de política y comparten un conjunto de creencias normativas que les proporcionan una base de valores para la acción social. Dichas creencias compartidas sirven a su vez de base para dilucidar las múltiples conexiones entre posibles cursos de política y resultados esperados. Asimismo, sus miembros comparten nociones de validación en su área de especialización, así como iniciativas políticas comunes, esto es, un conjunto de prácticas comunes asociadas con un 
conjunto de problemas hacia los cuales se dirige su competencia profesional. Además, estas comunidades buscan ejercer influencia sobre los decisores políticos a través de Track-Two-Diplomacy, procurando convencerlos acerca de la pertinencia de sus ideas, funcionando como linkage actors ${ }^{5}$ entre el nivel de la sociedad y el del Estado (Mingst 1995).

Para la consideración específica de la temática medioambiental en el contexto regional el enfoque constructivista parece adecuado dado que la ciencia funciona frecuentemente como portavoz del medioambiente. Así, en relación por ejemplo con los temas de contaminación ambiental, es evidente la capacidad de las comunidades epistémicas para modificar la percepción y formular el contexto para respuestas colectivas a problemas internacionales (Adler y Haas 1992). Por fuera de la academia, el medioambiente con frecuencia careció de voz propia, a la inversa de los intereses económicos y sociales expresados por el sector privado, los sindicatos u otras organizaciones sociales con representación en los parlamentos (Scholz 2007, 178). Además, la pluralidad y la calidad de las interfaces existentes entre la ciencia y la política son factores decisivos para incluir la dimensión ambiental de forma adecuada en el proceso político.

A la consideración de las comunidades epistémicas en términos de participación en la definición de la agenda medioambiental del Mercosur puede sumarse la referencia a las organizaciones de la sociedad civil. Cabe aquí mencionar aspectos comunes de la episteme compartida por ambos actores, que otorgan a las cuestiones medioambientales carácter prioritario. Se tematiza así el fenómeno frecuente en ALC donde el saber técnico supera los límites estrictos de la academia y se pone a disposición de los movimientos ambientalistas (Reboratti 2007, 170), en algunos casos incluso en la persona de los mismos expertos. Por otra parte esta consideración conjunta se ve justificada por un lado por la fluidez creciente de la interacción del saber técnico medioambiental con el saber consuetudinario en la materia, propio de determinados actores de la sociedad civil como las organizaciones indígenas.

\section{Situaciones nacionales}

Brasil

El caso de Brasil resulta especialmente interesante ya que explicita conflictos propios en torno a la soberanía nacional y la potestad de Estado para disponer de los recursos naturales a fin de sustentar el desarrollo económico ${ }^{6}$, por un lado,

5 Mingst (1995) categoriza como linkage actors a individuos, representantes gubernamentales y actores no estatales que actúan a través de las fronteras estatales ejerciendo influencia sobre las políticas públicas.

6 La Constitución de 1988 en su Capítulo VI, art. 225 consagra el derecho para todos «a un medio ambiente ecológicamente equilibrado, bien de uso común del pueblo y esencial para una sana calidad de vida, imponiéndose al Poder Público y a la colectividad el deber de defenderlo y preservarlo para las generaciones presentes y futuras", 
y la co-responsabilidad global en materia ambiental movilizada especialmente en torno de la Amazonia, por el otro. Son evidentes aquí las paradojas que enfrenta el Estado brasilero: cuanto más procura perfilarse como potencia con liderazgo regional, mayores son las expectativas de la comunidad internacional, por lo que el país se ve confrontado con la necesidad de asumir un rol pionero en Sudamérica y cumplir con estándares ecológicos y sociales. En cuanto al posicionamiento de Brasil en la arena global respecto a diversas temáticas medioambientales, destaca su liderazgo en energías renovables, en particular en biocombustibles, donde Brasil se ha posicionado activamente como gran exportador, convirtiendo además a los biocombustibles en punta de lanza de su política exterior.

A la inversa, la negativa a asumir compromisos en materia de desforestación y reducción de emisiones en conferencias internacionales revela los límites del modelo agroexportador. A eso se suman los intentos de modificar el Código Forestal, relativizando los compromisos asumidos en Copenhague en $2009^{7}$. Aunque en las negociaciones internacionales sobre cambio climático se destaca el nivel científico y diplomático de Brasil, revelando un potencial significativo para un ejercer un rol ejemplar en cuanto a política ambiental y social en ALC, en algunos casos de políticas medioambientales como el establecimiento de zonas de reserva ecológica, se hace evidente el conflicto con los grandes proyectos energéticos y de infraestructura del gobierno. Frecuentemente dichos proyectos, cuyos planes originarios se remontan a la política estratégica relativa a Amazonas de los años setenta y que han sido incluidos en el PAC (Programa de Acelação do Crescimiento), enfrentan el rechazo masivo de la sociedad civil: paradigmáticos son los enfrentamientos por la construcción de la represa de Belo Monte y el rol protagónico del Movimento Xingu Vivo Para Sempre (MXVPS), que aglutina a más de 250 organizaciones de base de la región amazónica (Peters 2011). En otros casos, como el de la lucha contra la contaminación atmosférica urbana, las organizaciones ambientalistas han desempeñado un rol más modesto de fiscalización, denuncia y propuesta de nuevos proyectos (Simioni 2003, 82).

Por su parte, los movimientos sociales así como las organizaciones de la sociedad civil del Brasil se caracterizan crecientemente por una articulación temática independiente del origen social de sus miembros, promoviendo la no violencia y orientándose al trabajo en red (Zimmering 2008, 40). Sus actividades están generalmente bien definidas, siendo las más significativas aquellas orientadas a la preservación de algún ecosistema, el mejoramiento de la calidad del medioambiente (aire, agua, residuos sólidos), la educación ambiental y la difusión de información sobre agricultura sostenible. Si bien el marco legal de sus acciones está dado por

señalando las incumbencias del Poder Público para asegurar dicho derecho. También cabe mencionar la Ley específica No 6.938 de 1981.

7 En el debate sobre la «modernização» del Código Forestal, la Sociedade Brasileira para o Progresso da Ciência (SBPC) y la Associação Brasileira de Ciências (ABC) presentaron en octubre 2011 un documento que rebate puntos polémicos de la propuesta aprobada por la Cámara Federal. 
su inclusión en la Constitución de 1988, la ley reglamentaria de la participación de la sociedad civil (Ley 9790/99) solo reconoce a las OSCIP (Organização da Sociedade Civil de Interesse Público).

En el caso de Brasil, los llamados «nuevos» movimientos sociales que surgen a partir de los setenta, reclaman al Estado mayor eficiencia, transparencia, control y obligación de rendir cuenta de sus actos (Reboratti 2007, 170; Peters 2011, 2), lo que puede interpretarse como el inicio de un cambio cultural y una reacción a la debilidad crónica del sistema de partidos políticos. Deben considerarse aquí los condicionamientos de la cultura política de ALC, marcada por tradiciones estatistas centralizadoras, patrimonialistas y determinadas por relaciones basadas en el clientelismo, la meritocracia y los intereses creados entre la sociedad y el Estado (Simioni 2003, 69). Así, las cuestiones planteadas por organizaciones ambientalistas se vinculan estrechamente con la construcción de ciudadanía para aquellos en situación de desigualdad, enfatizando los derechos sociales y las consecuencias de la degradación socioambiental.

En lo que hace a las comunidades epistémicas ambientales, estudios de casos indican que es necesaria una mirada diferenciada que evite reduccionismos propios del enfoque teórico elegido respecto a una eventual homogeneidad cognitiva y normativa. Así, por ejemplo, los miembros de la "comunidad epistémica climática» o "grupo $\mathrm{MCT}^{8}{ }^{8}$ presentan identidades más complejas, mayor ambivalencia, mayor capacidad de agencia y lealtades más selectivas frente a la «episteme climática» que lo que se asume desde la teoría, evidenciando una adhesión inestable, limitada e internamente fragmentada por parte de los científicos implicados. Mientras que por un lado estos especialistas se articulan en redes profesionales transnacionales, son también plenamente conscientes del impacto de la historia, la geografía y las realidades socio-económicas que se traduce en el predominio de los países desarrollados en los foros como el IPCC (Intergovernmental Panel on Climate Change), generando desconfianza en la academia brasilera. Por otra parte, dicho grupo, corporizando cierta tendencia a la convergencia normativa, ha impulsado progresivamente posiciones y políticas nacionales menos reactivas a la participación de Brasil en regímenes internacionales en materia climática, procurando mayor apertura y compromiso con el desarrollo de las ciencias ambientales (Lahsen 2004, 153 y ss.) Asimismo, su participación en el Fórum Brasileiro de Mudanças Climáticas junto a las instancias gubernamentales y a representantes de la sociedad civil facilita el trasvase de sus posiciones.

Por otra parte, la cuestión de la asignación de recursos a la investigación constituye un tema crítico ${ }^{9}$ (Lahsen 2004, 158.). Desde la óptica de la construcción social de la realidad, la investigación académica en materia medioambiental (en

8 Grupo afiliado al Ministerio de Ciencia y Tecnología (MCT) y de Itamaraty, encargado de establecer la posición brasilera en las negociaciones sobre cambio climático.

9 Es destacable el liderazgo brasilero en inversiones en ciencia y tecnología por ser el único país de ALC que destina un porcentaje superior al $1 \%$ del PBI a dicho rubro. 
particular la referida a la Amazonia) detenta debilidades significativas: una región que ecológicamente se encuentra en el centro de la atención mundial, no dispone de suficientes estructuras científicas propias a nivel local para trabajar problemas relevantes, ni para acompañar los procesos de transformación en curso. En el cuadro nacional, la situación real de la ciencia regional se condice con la marginalidad de la región dentro del contexto brasilero (Schönenberg 2007, 203-204).

Al mismo tiempo, la importancia creciente de la problemática ambiental durante los últimos años determinó la creación de centros de investigación y formación orientados a incidir en la definición de políticas públicas que incorporaron la temática medioambiental y en particular la sustentabilidad. Desde el ámbito privado se ha procurado un acercamiento técnico en instancias como el Centro de Estudios en Sustentabilidad (GVces) de la Escuela de Administración de Empresas de la Fundación Getúlio Vargas (FGV-EAESP) ${ }^{10}$ con foco en el nivel local, nacional e internacional. También destaca el Instituto Ethos de Empresas y Responsabilidad Social, creado en 1998 como polo de organización de conocimiento para apoyar a las empresas en una gestión social y medioambientalmente sustentable.

El desarrollo del agronegocio en la última década estimuló el surgimiento de instancias como el Instituto de Estudios de Comercio y Negociaciones Internacionales (ICONE). Asimismo, las entidades representativas de intereses privados han procurado desarrollar mecanismos de transferencia de tecnología y nuclean a sus asociados en proyectos de protección medioambiental y sustentabilidad. ${ }^{11}$ También han surgido entidades públicas como el Instituto de Investigaciones Ecológicas (IPÊ), PENSA (Centro de Conocimientos en Agronegocios), en el Departamento de Administración de la Facultad de Economía, Administración y Contabilidad de la Universidad de San Pablo o el Centro de Desarrollo Sustentable de la Universidad de Brasilia.

Respecto a los preparativos para Río+20, y más allá de las actividades de la Comisión Nacional ${ }^{12}$ encargada de organizar la Cumbre, el Consejo de Desarrollo Económico y Social en el ámbito de la Presidencia inició en 2010 un proceso consultivo que permitió firmar un Acuerdo con 70 entidades de la sociedad civil (sociales, ambientalistas, académicas, empresariales y sindicatos de trabajadores) en octubre de 2011. El proceso, que apuntaba a un posicionamiento conjunto, pretende no solo acompañar al gobierno nacional de cara a la Conferencia, sino también establecer un compromiso político con el desarrollo sustentable durante la Cumbre (CDES 2011).

10 Aquí se estableció la plataforma Empresas pelo Clima (EPC) para gestar una transición hacia una economía de bajo carbono.

11 Puede citarse el Consejo Empresarial Brasileiro para el Desarrollo Sustentable (CEBDS). A nivel sectorial la Unión de la Industria de la Caña de Azúcar (UNICA) ejecuta programas de capacitación para la cadena productiva sucro-energética sobre responsabilidad social empresarial y competitividad responsable. También pueden mencionarse actividades de la Asociación Brasilera de Celulosa y Papel (BRACELPA).

12 La Comisión Nacional de Brasil inició su tarea en julio de 2011 para entregar una propuesta a Naciones Unidas en noviembre. La Comisión encabezada por el ministro de Relaciones Exteriores y la ministra de Medio Ambiente incluye 18 ministerios, distintos sectores sociales y entidades gubernamentales. 


\section{Argentina}

Como argumentan Pelfini y Beling (2011, 3 y ss.), el caso argentino presenta algunas particularidades: claramente sin detentar las condiciones de potencia emergente asignadas a Brasil, por algunas de sus características sí podría atribuírsele el estatus de "sociedad emergente» por asumir circunstancialmente algún grado de liderazgo en algunos campos, más allá de sus aspiraciones constantes a alcanzar una posición de protagonismo. Más aún, por sus repetidos ciclos de stop and go, Argentina suele ser considerado como un eterno país emergente, aunque a la larga el eterno candidato corra el riesgo de eliminar dicho carácter por la pérdida de la excepcionalidad. Sin embargo, dos cualidades le aseguran un lugar destacable: por un lado, su creatividad cultural le permite ejercer algún liderazgo en Sudamérica, mientras que por otro su carácter de potencia agroalimentaria de primer orden la posiciona en forma privilegiada en el mercado de las commodities, mientras que le proporciona las condiciones para consolidarse como proveedor de biocombustibles. Sin embargo, dados los problemas de sustentabilidad social y ambiental del modelo de agronegocios, difícilmente puede generarse el tipo de innovación requerida para convertir a la Argentina en una sociedad emergente en cuestiones ambientales y energéticas. Aún así, existiría espacio para una novedosa conjunción de creatividad cultural e innovación productiva en el sector agropecuario que podría generar las condiciones para una auténtica emergencia capaz de sustentar un liderazgo en un rubro clave como el de las energías renovables no convencionales. La clave radicaría en una articulación novedosa entre el nivel macro (definición de reglas jurídicas y económicas, así como de políticas de fomento de ciencia y tecnología por parte del Estado) ${ }^{13}$, el nivel meso (mercado asociado a la innovación productiva) y el nivel micro (sociedad civil). Precisamente es a nivel de la sociedad civil donde encuentra especial expresión la creatividad cultural mencionada, siendo posible identificar una vitalidad particular, abierta a la experimentación y de relación crítica con la autoridad, con cierta propensión a la radicalización por lo que con frecuencia devendría más reactiva que proactiva.

$\mathrm{Al}$ respecto debe señalarse que los movimientos ambientalistas desarrollados en Argentina desde los años noventa con frecuencia surgieron a causa de conflictos en torno a proyectos mineros, instalación industrial, contaminación, deforestación o proyectos de aprovechamiento hidrológico; siendo originalmente movimientos sociales de base, muchos han volcado con posterioridad sus reivindicaciones al tema ambiental. Se registra así un incremento de organizaciones ambientalistas, entre las que destacan algunas surgidas por iniciativa de técnicos y profesionales preocupados por la promoción de una agenda de sustentabilidad, tales como el Programa

13 La Constitución Nacional en su art. 41 establece el derecho a gozar de un ambiente sano, equilibrado, apto para el desarrollo humano y para que las actividades productivas satisfagan las necesidades sin comprometer las de las generaciones futuras, por lo que fija el deber de preservarlo. Dentro del marco reglamentario destaca la Ley General del Ambiente No 25.675 de 2002. 
Argentina Sustentable (PAS), Bios ONG, Los Verdes, Pro Eco Grupo Ecologista, Alihue, Greenpeace, Vida Silvestre, Taller Ecologista, Fundación Ambiente y Recursos Naturales (FARN), Amigos de la Tierra, así como la Asociación Argentina de Abogados Ambientalistas, que desarrollaron cierta capacidad de articulación y alcanzar posicionamientos conjuntos frente a políticas nacionales sectoriales proponiendo una agenda alternativa. ${ }^{14}$

Entre las comunidades epistémicas, es posible identificar instituciones públicas y privadas abocadas específicamente a la investigación, formación y eventualmente diseño de políticas públicas en materia medioambiental en diferentes áreas temáticas. Dentro del primer grupo puede mencionarse el Centro Tecnológico para la Sustentabilidad (CTS) de la Universidad Tecnológica Nacional, las diferentes líneas de trabajo de las Universidades de San Martín, de Rosario y de Buenos Aires, y el Instituto de Ecología y Desarrollo Sustentable (INEDES) de la Universidad de Luján. También las instituciones públicas han incorporado líneas relativas al desarrollo sustentable, protección del medioambiente y energías renovables, como el INTI (Instituto Nacional de Tecnología Industrial) ${ }^{15}$ o el INTA (Instituto Nacional de Tecnología Agropecuaria).

Entre las entidades privadas pueden mencionarse el Centro de Investigación, Observación y Monitoreo Territorial y Ambiental (CIOMTA) en Santa Fe, así como el Centro de Estudios de Energía, Política y Sociedad (CEEPYS). También cabe identificar instituciones de investigación y formación focalizadas en diversas áreas que han incorporado la temática medioambiental y energética a sus líneas de trabajo, tales como la Fundación de Investigaciones Económicas Latinoamericanas (FIEL) y la Facultad Latinoamericana de Ciencias Sociales (FLACSO Argentina).

También los intereses sectoriales han buscado posicionarse en clave «ambiental» aunque su involucramiento pareciera menor que el de sectores similares en Brasil, donde, como reacción al avance de las exigencias del marco normativo, muchas organizaciones sectoriales incorporan explícitamente la sustentabilidad ambiental como línea de trabajo. En el caso de las organizaciones industriales o agropecuarias argentinas como la Unión Industrial Argentina (UIA), la Sociedad Rural Argentina (SRA) o las Confederaciones Rurales Argentinas (CRA) pueden identificarse actividades puntuales de asesoramiento o difusión de información o estudios. Con mayor grado de especialización cabe mencionar actividades de asesoramiento y capacitación brindada a sus miembros por la Cámara Empresaria de Medioambiente (CEMA), la Cámara Argentina de Energías Renovables (CADER) y la Cámara Argentina de Biocombustibles (CARBIO).

14 En septiembre de 2011 algunas de las organizaciones mencionadas se pronunciaron conjuntamente de forma crítica frente al anuncio del gobierno argentino de profundizar el Plan Estratégico Agroalimentario Industrial, señalando prioridades para la política ambiental nacional (COM Ambiental, 27/9/11).

15 El INTI desarrolló una división especial referida a huella de carbono y es miembro del think tank internacional Global Ecological Footprint. 


\section{Perspectivas}

El análisis del contexto global permite aseverar que la gobernanza multilateral actual no dio respuesta eficaz al desafío de lograr una mayor coherencia entre las condiciones que resultan de los mecanismos y foros globales existentes y las necesidades reales de los países de ALC (CEPAL 2011, 234), lo que es particularmente evidente en materia de desarrollo sustentable. También la consideración de la evolución del tratamiento gubernamental de las cuestiones medioambientales en los países de ALC proporciona claves para interpretar las dificultades para consensuar posiciones como base de su proyección externa. En temas clave como el cambio climático, ALC no se presenta a las negociaciones internacionales en forma articulada. Dado que los impactos de dicha problemática en la región son muy variados, las propuestas efectuadas por algunos países carecen de coordinación y son el resultado de sus intereses específicos, con diferentes prioridades y diversidad de agendas (FuentesBracamontes 2011). Dicha desarticulación tiene costos potenciales en términos de oportunidades perdidas (Acquatella 2008, 105-106): una estrategia de posicionamiento activo le permitiría captar beneficios del esfuerzo internacional al atraer recursos financieros y tecnológicos con costo relativamente bajo.

Frente a Río+20, y ante al reconocimiento de las ventajas que podría aparejar el establecimiento de acuerdos regionales (así como globales) basados en indicadores ambientales de desarrollo sostenible acordados previamente (CEPAL 2011, 238), la región de América Latina y el Caribe no puede correr el riesgo de asistir a la conferencia sin una hoja de ruta consensuada. Los esfuerzos y las discusiones en el marco de la CEPAL, tales como la Reunión Regional Preparatoria de las Naciones Unidas en septiembre de 2011, constituyen aportes en ese sentido, aunque resulta ilustrativo que documentos clave solo se refieran a políticas y acciones nacionales, sin abordar los esquemas de integración regional.

En el caso del Mercosur es evidente que, pese al enfoque diferencial conceptualizado como regionalismo estratégico, pese a la institucionalidad en materia medioambiental y pese a las intenciones explicitadas oportunamente, el bloque no consigue desarrollar una estrategia de posicionamiento y negociación externa que salga al encuentro de sus necesidades de desarrollo sustentable. A la heterogeneidad ambiental se suma la fragmentación del territorio sudamericano, donde las fronteras políticas pesan más que los límites ambientales (Reboratti 2007, 164-165). Puntualmente, la conflictividad por un tema medioambiental, como en el caso del conflicto entre Argentina y Uruguay por las papeleras exacerbó posturas nacionalistas. Asimismo, la escasa coherencia de las políticas internas ofrece claves para explicar las dificultades de la articulación de posiciones conjuntas en materia medioambiental, que de ser superadas permitirían una proyección asertiva del bloque y favorecerían una participación activa en los regímenes internacionales del caso. 
Mientras el caso brasilero permite identificar avances interesantes, el caso argentino evidencia ciertas dificultades de las comunidades epistémicas y la sociedad civil para actuar como linkage actors e influenciar el espectro de políticas públicas, incluida la política exterior. De cara al futuro cabe esperar la profundización del cambio progresivo de los últimos años en términos de movilización social. De hecho, las pautas tradicionales, estatistas y patrimonialistas de la práctica política tanto en Argentina como en Brasil no han constituido un impedimento para el surgimiento de diversas formas de participación de los sectores populares.

En lo que hace a la circulación de ideas, por su gran potencial de activación, las organizaciones de la sociedad civil estarían en situación de profundizar el cambio, aunque también pueden impedirlo o hacerlo retroactivo, lo que es significativo dado el énfasis en cuestiones socioambientales desde los años noventa. Sobre el rol de las comunidades epistémicas como portadoras de ideas y como agencias gestoras del cambio a través del impulso a la convergencia normativa y cognitiva, debe mencionarse su potencial de agenda-setters que enfatizan la centralidad de la problemática medioambiental como condición para un desarrollo sustentable. Para la plena consecución de esa función, la academia debiera superar bloqueos propios de su actuación. Al mismo tiempo, el análisis de su rol debe evitar reduccionismos propios del enfoque constructivista para dar cuenta de la diversidad de los intereses complejos y contradictorios de los múltiples sectores involucrados en los procesos de desarrollo, que buscan asimismo su legitimación y posicionamiento en clave "epistémica».

Respecto a los aportes de la sociedad civil y las comunidades epistémicas para definir una agenda externa medioambiental a nivel de Mercosur, su débil articulación a través de las fronteras no proporciona bases adecuadas para alcanzar posiciones conjuntas, lo que no quita mérito a las iniciativas existentes. Ocasiones puntuales como los preparativos para las Cumbres pueden ofrecen coyunturas favorables para acciones coordinadas y permiten un prudente optimismo. En otro nivel, la concreción la Agencia Ambiental del Mercosur podría facilitar el diseño de una agenda externa inclusiva si no se limitara a una iniciativa simbólica.

De cara a Río+20, la Reunión de Ministros de Medio Ambiente del Mercosur planteó la necesidad de posicionamientos conjuntos, paralelamente a los preparativos que los comités nacionales llevan adelante buscando la participación de la sociedad civil, donde es notoria la movilización alcanzada en Brasil en contraste con los otros países del bloque. Sin embargo, dada la participación de Brasil como anfitrión, los países del Mercosur debieran incrementar esfuerzos si pretenden optimizar la oportunidad para una proyección externa asertiva en la materia. La Cumbre ofrece asimismo una oportunidad para la consolidación del liderazgo de Brasil como potencia emergente con capacidad de incidir activamente sobre uno de los regímenes internacionales claves. Los otros países miembros del Mercosur debieran propiciar un avance 
en ese sentido, en el entendimiento que ello contribuye a sustentar en el futuro un regionalismo que realmente pueda reclamar para sí el rótulo de estratégico.

\section{Referencias bibliográficas}

Acquatella, Jean (2008) Energía y cambio climático: oportunidades para una política energética integrada en América Latina y el Caribe. Santiago de Chile: CEPAL.

Adler, Emanuel; Haas, Peter (1992) «Conclusion: epistemic communities, world order, and the creation of a reflexive research program», en International Organization. Vol. 46. No 1. Winter 1992, pp. 367-390.

Bizzozero, Lincoln (2009) «Integración regional en el Cono Sur 1980-2007. MERCOSUR como respuesta estratégica», en Guerra Borges, Alfredo (Ed.) Fin de época: de la integración tradicional al regionalismo estratégico. México: Siglo XXI.

Bizzozero, Lincoln (2011a) «América Latina a inicios de la segunda década del siglo XXI: entre el regionalismo estratégico y la regionalización fragmentada", en Revista Brasileira de Política Internacional. Año 54. No 1, pp. 29-43.

Bizzozero, Lincoln (2011b) «Aportes del MERCOSUR al regionalismo y a la teoría política de la integración regional: una mirada desde los veinte años del proceso», en Meridiano 47. Vol. 12. No 125. Instituto Brasileiro de Relaçōes Internacionais, pp. 4-10.

Briceño Ruiz, José (2006) «Regionalismo estratégico e interregionalismo en las relaciones externas del MERCOSUR», en Revista Aportes para la Integración Latinoamericana. Año XII. No 15, diciembre, pp. 28-42.

CDES (2011) Contribuições para a Conferência Rio+20. Documento conjunto «Acordo para o Desenvolvimento Sustentável». Brasilia.

CEPAL (2011) La sostenibilidad del desarrollo a 20 años de la Cumbre de la Tierra: avances, brechas y lineamientos estratégicos para América Latina y el Caribe. Versión preliminar. Santiago de Chile: CEPAL.

Colacrai, Miryam (1998) «La búsqueda de armonización ambiental en el MERCOSUR», en La política exterior argentina 1994-1997. Rosario: Ediciones CERIR, pp. 303-317.

COM Ambiental (2011) Agua y energía: áreas prioritarias para una política ambiental nacional. <http:/www.comambiental.com.ar/2011/09/agricultura-y-energia-areas.html>. Disponibilidad: $23 / 12 / 2011$

Fuentes Bracamontes, Rolando (2011) Impactos, políticas y posiciones de los países latinoamericanos rumbo a COP 17. ARI 130/2011. Real Instituto Elcano. 15/09/2011.

Guerra Borges, Alfredo (2009) Fin de época: de la integración tradicional al regionalismo estratégico. México: Siglo XXI.

Haas, Peter (1992) «Introduction: Epistemic Communities and International Policy Coordination», en International Organization. Vol. 46. No 1, pp. 1-35. 
Hänggi, Heiner (1998) «Sozialer Konstruktivismus als goldener Brücke? Zur jüngsten Theoriedebatte in der Disziplin der Internationalen Beziehungen», en Bulletin der Schweizerischen Akademie der Geistes- und Sozialwissenschaten. 1/98, pp. 32-41.

Koslowski, Rey; Kratochwil, Friedrich (1994) «Understanding Change in International Politics: The Soviet Empire's Demise and the International System», en International Organization. Vol. 48. No 2, pp. 215-247.

Lahsen, Myanna (2004) «Transnational locals: Brazilian Experiences of the Climate Regime», en Jasanof, Sheila/Long Martello, Marybeth (Eds.) Earthly Politics. Local and Global in Environmental Governance. Cambridge/Massachusetts: MIT Press, pp. 151-172.

Martínez Artega, Manuel (2011) Nexo entre medio ambiente y comercio en el MERCOSUR. Un camino posible y necesario hacia el desarrollo sustentable. Memoria de Grado para la obtención del título de Licenciado en Negocios Internacionales e Integración. Montevideo: Universidad Católica del Uruguay Dámaso Larrañaga.

Mercado Común del Sur (1991) Tratado para la constitución de un Mercado Común entre la República Argentina, la República Federativa del Brasil, la República del Paraguay y la República Oriental del Uruguay. Asunción.

MERCOSUR/CMC/Dec. No 26/03. Programa de Trabajo del MERCOSUR 2004-2006.

Mingst, Karen (1995) «Uncovering the Missing Liks: Linkage Actors and Their Strategies in Foreign Policy Analysis», en Neack, Laura/Hye, Jeanne/Haney, Patrick (Eds.) Foreign Policy Analysis. Continuity and Change in Its Second Generation. Englewood Cliffs.

Pelfini, Alejandro/Beling, Adrián (2011) "Argentina: paradojas de un eterno emergente», en Pelfini, Alejandro/Fulquet, Gastón, Beling, Adrián (Comp.) La Energía de los emergentes. Innovación y cooperación para la promoción de las energías renovables en el Sur global. Buenos Aires: FLACSO Argentina (próxima edición).

Peters, Inna (2011) Der Belo Monte Staudamm: Paradebeispiel für eine erfolgreiche Zivilgesellschaft in Brasilien? GIGA Focus Lateinamerika. 9/2011. Hamburg.

Reboratti, Carlos (2007) "América del Sur, Brasil y la cuestión ambiental», en Costa, Sergio/ Sangmeister, Hartmut/Steckbauer, Sonja (Orgs.). O Brasil na América Latina. Interações. Percepcões. Interdependências. São Paulo: Fundación Heinrich Böll/Anna Blume/ADLAF, pp. 163-176.

Scholz, Imme (2007) «Entre compromisos globais e intereses nacionais: elementos definidores da agenda da cooperação internacional na Amazônia brasilera", en Costa, Sergio/Sangmeister, Hartmut/Steckbauer, Sonja (Orgs.) O Brasil na América Latina. Interações. Percepcões. Interdependências. São Paulo: Fundación Heinrich Böll/Anna Blume/ADLAF, pp. 177-200.

Schönenberg, Regine (2007) «A ciencia na Amazônia brasilera: um potencial para o futuro da região?», en Costa, Sergio/Sangmeister, Hartmut/Steckbauer, Sonja (Orgs.) O Brasil na América Latina. Interaçôes. Percepcóes. Interdependências. São Paulo: Fundación Heinrich Böll/Anna Blume/ADLAF, pp. 201-213.

Secretaría de Medioambiente (2011) [http://www.ambiente.gov.ar/archivos/web/MERCOSUR/ file/XII\%20REUNION\%20DE\%20MINISTROS/RMMAM_2010_ATA02_ANE05_PT_ DesafiosCancua_Argentina\%20_2_.pdf] Disponibilidad: 23/12/2011. 
Secretaría MERCOSUR (2006) Medioambiente en el MERCOSUR. Relevamiento No 001/06. Montevideo, 20 de febrero de 2006. [http://www.mercosur.int/innovaportal/ file/736/1/medioambienteenelmercosur.pdf] Disponibilidad: 23/12/2011.

Simioni, Daniela (2003) Contaminación atmosférica y conciencia ciudadana. Santiago de Chile: CEPAL.

Zimmering, Raina (2008) «Revolution in der Demokratie? Neue soziale Bewegungen in Lateinamerika», en WelTrends. No 61, pp. 35-41.

Recibido el 23 de diciembre de 2011

Aceptado el 29 de marzo de 2012

\section{Resumen}

El artículo analiza la búsqueda de posiciones comunes del Mercosur Ambiental, teniendo en cuenta la evolución del proceso regional. Desde el constructivismo social se pretende identificar las contribuciones de las comunidades epistémicas y de la sociedad civil para definir una agenda ambiental para Brasil y Argentina, y se describen algunas perspectivas.

Palabras clave: agenda ambiental; Argentina; Brasil; Mercosur.

\section{Abstract}

The paper reviews the pursuit of joint environmental positions within Mercosur considering the evolution of the regional process. Based on social constructivism it seeks to identify contributions of epistemic communities and civil society to define an environmental agenda for Brazil and Argentina and outlines some perspectives.

Keywords: environmental agenda; Argentina; Brasil; Mercosur. 